Journal of Case Reports 2018;8(3):172-175

\title{
Successful Management of Cardiac Arrest Following Cobra Bite: A Case Report from a Rural Critical Care Unit in India
}

\author{
Harish Handyal ${ }^{1}$, Gopi Kumar ${ }^{1}$, Ketty E. Arce ${ }^{2}$, Praveen Kumar Birru ${ }^{3}$ \\ Departments of ${ }^{1}$ Critical Care, ${ }^{2}$ Emergency Medicine, and ${ }^{3}$ Orthopedics; RDT hospital, Bathalapalli, Anantapur:515661, India.
}

\section{Corresponding Author: \\ Dr. Harish Handyal \\ Email: drharry69@gmail.com}

This is an Open Access article distributed under the terms of the Creative Commons Attribution License (creativecommons.org/ licenses/by/3.0).

Received : April 30, 2018

Accepted : July 5,2018

Published : July 15, 2018

\begin{abstract}
Background: Neuro-paralysis leading to type II respiratory failure is the most important cause of morbidity and mortality in victims bitten by venomous snakes of Elapidae family. In-hospital cardiac arrests are secondary to presumed acute respiratory compromise and an excellent outcome can occur after well-choreographed, high quality cardio-pulmonary resuscitation. Compartment syndrome is characterized by an increase in intra-compartmental pressure with subsequent neurovascular compromise and tissue necrosis. Hence early necrectomy and debridement are indicated in such instances. Case Report: We herewith report a case of snake bite with cardiac arrest following severe neurotoxic envenomation, was managed successfully in our critical care. In our case, the presentation was late, resulting in a substantial delay in anti-venom therapy. The resultant delay contributed to a significant morbidity and prolonged hospital admission. Conclusion: The case report illustrates some practical difficulties faced by clinicians pertaining to complications. Institution of appropriate therapy without delay results in better outcome, including in a rural critical care setting.
\end{abstract}

Keywords: Cardiac Arrest, Respiratory Paralysis, Compartment Syndrome, Snake-Bite Envenomation, Anti-Snake Venom (ASV).

\section{Introduction}

Snake bite is a significant health concern, especially in rural populations of India. High mortality is due to poor health services in rural areas and delay in getting the victim to a well-equipped health care facility, where anti-snake venom can be administered [1,2]. A nationally representative snake bite mortality survey in India has highlighted 45,900 deaths annually, with the highest mortality rate in the state of Andhra Pradesh [1].

Snake venom is no single toxin but a cocktail of many components like enzymes, polynucleotide toxins, non-toxic proteins, carbohydrates, metals, lipids, free amino acids, nucleotides and biogenic amines. In most serious cases the envenomed victim may present in organ and/or system failure, muscle paralysis, respiratory failure, hemodynamic instability, acute kidney injury, coagulation disorder (venom-induced consumption coagulopathy, VICC) and severe local injury due to necrosis and compartment syndrome. Severe envenomation and its serious complications requiring organ and systems support should be treated in the intensive care setting $[2,3,4]$. We herewith report a case of snake bite which developed cardiac arrest following severe neurotoxic envenomation was managed successfully in our critical care.

\section{Case Report}

A 50-year-old male presented to the emergency department with history of cobra snake bite. Bite to presentation time was 6 hours due to delay in transporting the patient from a remote 
village and also due to delay in referral from the primary healthcare center. On examination he was unconscious (GCS: 7/15), restless with severe tachypnea (respiratory rate: 48/min) and tachycardia (heart rate: $140 / \mathrm{min}$ ) with hypertension (blood pressure: 160/98 mmHg). Local examination showed swelling over the site of bite (lateral aspect of left forearm) with two fang marks. In view of rapidly worsening respiratory distress, patient started to desaturate and he developed witnessed cardiac arrest (bradycardia followed by asystole). Cardiopulmonary resuscitation started as per advanced cardiovascular life support (ACLS) guidelines and return of sinus rhythm with return of spontaneous circulation (ROSC) achieved within 3 minutes. He was hemodynamically optimized and shifted to intensive care unit (ICU).

His initial investigations showed, hemoglobin: $14.6 \mathrm{gm} \%$ and total counts: 17600/ $\mathrm{mm}^{3}$. His liver function tests were deranged with AST: 1850, ALT: 400. CPK \& LDH were high (1420 \& 4880 respectively). His coagulation profile was normal. 2D echo (FATE) showed global hypokinesia and poor left ventricular function with ejection fraction: $30-35 \%$. His arterial blood gas analysis with $100 \%$ oxygen revealed metabolic acidosis with hyper-lactatemia $(\mathrm{pH}$ : 7.01, base excess of $-12 \mathrm{meq} / \mathrm{L}$, bicarbonate of $18 \mathrm{meq} / \mathrm{L}$ and serum lactate of $65 \mathrm{mg} \%$ ).

In the ICU, patient was started on lung protective mechanical ventilation. Invasive lines secured to start vasopressor and inotropic supports and post-cardiac arrest care bundle was initiated. He was started on anti-snake venom (ASV) therapy according to protocol (initial dose of 10 vials of polyvalent ASV followed by one vial per hour continuous infusion). Next 2 days, patient continued to be mechanically ventilated. He was hemodynamically stable, left ventricular contractility improved and vasopressor and inotropic requirements weaned and stopped and he was gradually rewarmed passively from second day.
Patient developed increasing swelling followed by acute compartment syndrome of the left upper limb [Fig.1] for which he underwent emergency fasciotomy on $3^{\text {rd }}$ day [Fig.2] and was taken up for repeated debridement (3 times), between $5^{\text {th }}$ to $10^{\text {th }}$ day. He underwent tracheostomy in view of prolonged need for mechanical ventilation on day 8 . Subsequently, he was gradually weaned off from ventilator and tracheostomy decannulated on $14^{\text {th }}$ day and patient was later shifted to ward on $16^{\text {th }}$ day.

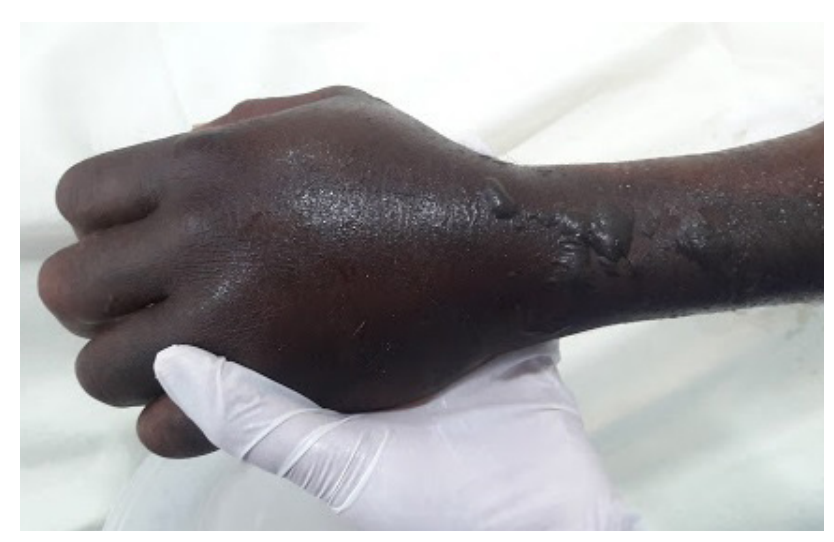

Fig.1: Compartment syndrome of left forearm.

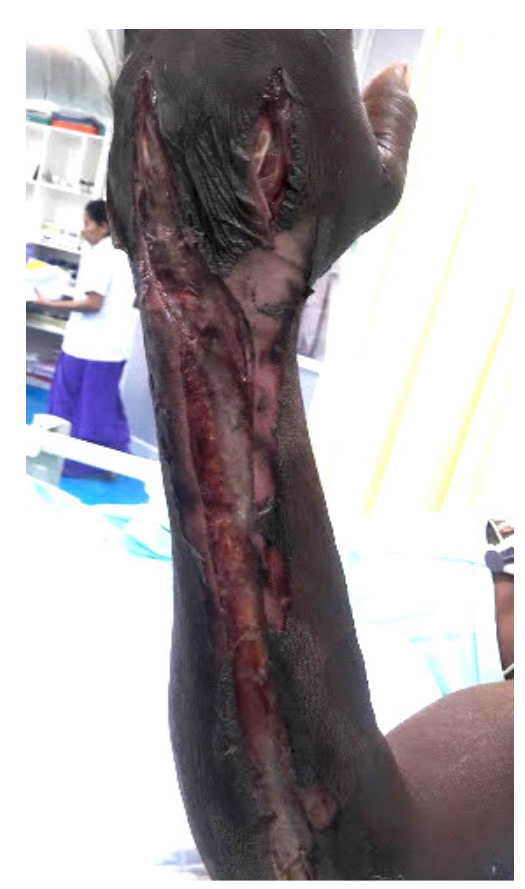

Fig.2: Post-fasciotomy wound. 


\section{Discussion}

Snake bite is a significant health concern, especially in rural populations of tropical and subtropical countries. In India, snake bites take a heavy toll of human lives, and therefore warrant urgent attention [1,2]. Cobra venom consists of three types of proteins: cardiotoxin, neurotoxin, and phospholipase A2. The neurotoxin of cobra mainly affects muscles of the eye, tongue, throat \& chest causing respiratory failure. The primary action of cardiotoxin is directly on cell membrane, causing many effects on the skeletal, cardiac, smooth muscles, nerves and neuromuscular junctions, thus contributing to circulatory and respiratory paralysis and cardiac asystole [5].

In-hospital cardiac arrests are secondary to presumed acute respiratory compromise and/ or circulatory shock, with predictable progressive deterioration before the event. Excellent outcomes can occur after well-choreographed, high quality CPR. In our patient, cardiac arrest was due to cardiotoxicity and severe neurotoxic envenomation, which was promptly recognized and high quality CPR was instituted with good neurological outcome $[6,7]$.

Effective post-cardiac arrest care consists of identification and treatment of the precipitating cause of cardiac arrest combined with the assessment and mitigation of ischemia-reperfusion injury to multiple organ systems. Benefit is possible with appropriate forms of early goal-directed therapy and achieving therapeutic hypothermia within the first few hours, followed by gradual rewarming and ensuring glycemic control. Creation of a bundle that incorporates these various aspects of care would more likely ensure that most patients recover with optimal neurological function [8]. In our patient we strictly adhered to the post-cardiac arrest bundle for 24 hours with positive outcome.

Timely administration of ASV along with cardio-respiratory support is the only effective treatment available for neurotoxic snake bite.

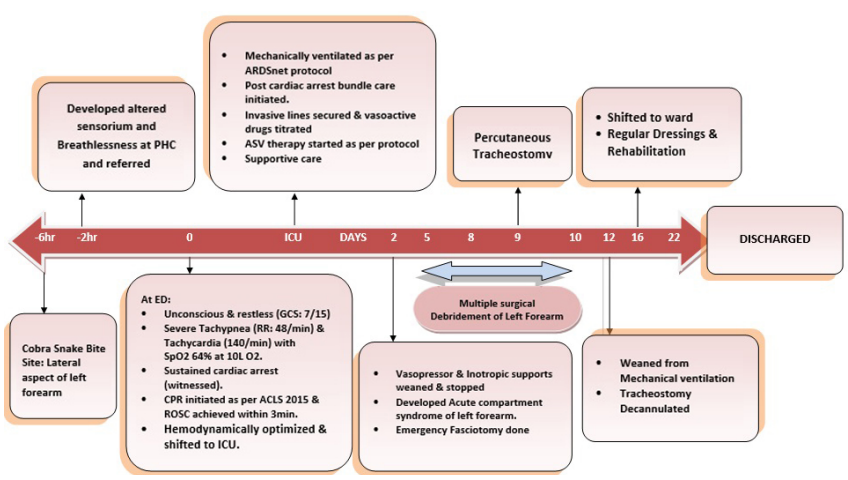

Fig.3: Timeline of clinical events in the hospital.

ASV is the most effective when administered early enough to neutralize venom in the circulation before it reaches the target site [2,9]. However, there is no universal consensus on the optimal dose and protocol of ASV administration [10]. In our patient, there was an inordinate delay and he had not received ASV prior to our hospital admission. A total of 58 vials of ASV were administered in our critical care over a period of 48 hours.

Neuroparalysis leading to type II respiratory failure is the most important cause of morbidity and mortality in victims bitten by venomous snakes of Elapidae family. Severity of envenomation and progression to respiratory failure is related to several factors such as dose of venom injected, potency of venom, anatomic location of bite, age, health, size and possibly immune status of victim, and the nature and timely intervention $[2,4,9]$. In all such patients, timely administration of ASV and institution of cardio-respiratory support is associated with an excellent outcome $[3,4]$. It is concluded mechanical ventilatory support is a lifesaving procedure in patients of snake bite manifestation signs of bulbar and respiratory paralysis and timely institution can prevent hypoxic injury to brain [5]. Our patient presented with severe envenomation in respiratory paralysis and needed prolonged ventilatory support for 15 days.

Compartment syndrome is characterized by an increase in intra-compartmental pressure 
with subsequent neurovascular compromise and tissue necrosis. It occurs due to a vicious cycle of edema causing hypoxia and acidosis, which further increases capillary permeability and fluid extravasation. This results in a volume increase in the closed fascial compartment, which ultimately compromises circulation and causes irreversible muscle and nerve damage $[2,5]$. Even if the antivenom treatment does not have essential influence on the local tissue disability, its administration may reduce the extent of necrosis. Hence early necrectomy and debridement are indicated in such instances [12]. Our subject received early and appropriate care but progressed to develop compartment syndrome of the left upper limb after two days of admission for which he had to be taken to the operating theatre for emergency fasciotomy. The timely fasciotomy and multiple debridement in our patient helped the patient achieve excellent functional results.

\section{Conclusion}

There is a pressing need to educate the public about the hazards of snakebite and early hospital referral will decrease mortality and morbidity. A national policy should be formulated and implemented to ensure prompt availability and effective use of ASV in the rural areas. Administration of antisnake venom and cardio-respiratory support is the mainstay of therapy in neurotoxic envenomation with respiratory failure. It is essential to diagnose acute compartment syndrome promptly and perform urgent fasciotomy to prevent permanent tissue necrosis. In case of cardiac arrest, follow ACLS guidelines for CPR and strictly adhere to post-arrest care bundle. Institution of appropriate therapy without delay results in better outcome, including in a rural critical care setting.
Contributors: HH: manuscript writing, patient management; GK: manuscript editing, patient management; KEA, PKB: critical inputs into the manuscript. HH will act as guarantor. All authors approved the final version of this manuscript.

Funding: None; Competing interests: None stated.

\section{References}

1. GuptaYK, Peshin SS. Snake bite in India: Current scenario of an old problem. J Clin Toxicol. 2014;4:1000182.

2. Mithra S. Snake bite in India \& its management. J Indian Medical Assoc. 1987;85:129-131.

3. Agarwal PN, Aggarwal AN, Gupta D, Behera D, Prabhakar S, Jindal SK. Management of respiratory failure in severe neurotoxic snake envenomation. Neurol India. 2001;49:25-28.

4. Valenta J, Stach Z, Michálek P. Severe snakebite envenoming in intensive care. Prague Med Rep. 2016;117:153-163.

5. Warrell, David A. Snake bite. The Lancet, Volume 2010;375:77-88.

6. Steven L. Kronick et al. American Heart Association Guidelines Update for Cardiopulmonary Resuscitation and Emergency Cardiovascular Care. Circulation. 2015;132(suppl 2): S397-S413.

7. Callaway CW. Post-cardiac arrest care: 2015 American Heart Association guidelines update for cardiopulmonary resuscitation and emergency cardiovascular care. Circulation. 2015; 132(suppl 2): S465-S482.

8. Anantharaman V. The post-resuscitation bundle. Singapore Med J. 2011;52:607.

9. Harsoor SL, Gurudatta CL, Balabhaskar S, Kiranchand $\mathrm{N}$, Bhosale R. Ventilatory management of patients with neuroparalytic envenomation. Indian J Anaesth. 2006;50:452-455

10. Agrawal A, Gupta A, Khanna A. What dose of anti-snake venom should be given in severe neuroparalytic snake bite? Annals of Thoracic Medicine. 2011;6(1):47-48.

11. Akram S, Ishaque $M, A b b a s ~ S$. Mechanical ventilation in snake bite. Pak Armed Forces Med J. 2004;54:282-286.

12. Dhar D. Compartment syndrome following snake bite. Oman Medical Journal 2015;30:146-146. 